menées: établissement de statistiques démographiques et agricoles, évaluation de la consommation et des niveaux de vie. Participeront à cette mission MM. Gillot (Service des Statistiques), Libercier et Berger (Service de l'Agriculture), Périssé (ORSTOM). La seconde mission, celle du CSRSOM, permettra à M. Pauvert d'étendre ses recherches aux problèmes posés par l'émigration kabrè dans le sud-ouest du Togo et la Gold-Coast: relations entre les immigrés et leur groupe d'origine, relations entre les immigrés et les groupes autochtones.

En liaison directe avec M. Pauvert, M. Périssé a exécuté une enquête alimentaire dans deux groupements kabrès du Centre-Togo, l'un d'implantation ancienne, l'autre d'implantation récente, et une enquête de même type en pays kabrè d'origine. Elles serviront de point de départ aux enquêtes plus étendues que M. Périssé doit réaliser dans le cadre de la Mission du Service des Statistiques.

2. A l'Institut de Recherches du Cameroun, M. Diziaine, géographe, a poursuivi ses recherches sur la population de Douala et la région de Yaoundé. Il achève, en collaboration avec le Directeur de l'Institut, $M$. Bonnet-Dupeyron, la mise au point des cartes d'élevage et de densité de population, qu'ils avaient entreprises lors d'une précédente mission, au Soudan français.

3. M. Soret, de l'Institut d'Études Centrafricaines (Brazzaville) poursuit l'exécution des cartes ethno-démographiques de l'A.E.F. au 1.000.000 par points. Les feuilles Sud (Brazzaville et Pointe Noire) ont paru. Les feuilles du Gabon sont à l'étude.

4. A Madagascar M. Molet va faire paraître sa thèse de doctorat sur le Baia de la Reine et la démographie des Tsimihety. Il poursuit l'étude des migrations tsimihety, population en voie d'extension démographique notable dans la partie nord du plateau.

IV. Le colonel Chédeville poursuit en Côte Française des Somalis l'enquête linguistique et sociologique qui lui a été confiée par l'Institut International Africain sur les Afar (Danakil).

M. Pepper, musicologue de l'ORSTOM (Institut de Recherches Centrafricaines) a ramené en France de nombreux documents sur la mode d'expression sonore des noirs du Congo. Il a effectué une tournée de conférences aux États-Unis. Actuellement à Paris, il prépare trois volumes accompagnés de disques qui donneront les résultats de ses observations pendant quinze ans.

Un linguiste, M. Jacquot, qui avait précédemment participé à la mission de délimitation des langues bantoues de l'Institut International Africain, a été engagé par l'ORSTOM et affecté à Brazzaville.

\title{
La Semaine scolaire de l'Union Franfause
}

LA semaine scolaire de l'Union française, présidée par M. Albert Sarraut, président de l'Assemblée de l'Union française, et patronnée par les Ministres de l'Education nationale et de la France d'Outre-Mer, s'est terminée le 17 mars par un vaste concours dans tous les établissements publics d'enseignement de la métropole et des territoires d'outre-Mer.

L'année dernière déjà, le Comité de la Journée scolaire de l'Union française, créé à l'initiative de l'éminent ethnologue Marcel Griaule, mort récemment, et de Mme Malroux, Conseillers de l'Union française, et présidé par M. Albert Sarraut, avait organisé un concours similaire. 300.000 jeunes gens at jeunes filles avaient été candidats et les lauréats avaient gagné soit de faire un voyage outre-mer pour ceux de la métropole, soit pour ceux de l'outreMer, de se réunir avec leurs camarades de la métropole en un séjour commun d'un mois sur la Côte Basque.

Devant ce succès, l'initiative a été réitérée cette année par le Comité de la Journée scolaire dont les buts ont été définis au cours d'une conférence de presse par M. André Chimier, Secrétaire général de l'Assemblée de l'Union française, représentant $M$. Sarraut:

' Créer entre les jeunes de tous les pays, de toutes les races, de toutes les religions, qui 
composent la population scolaire de l'Union française, un lien intellectuel en même temps que le sentiment de l'appartenance à une même communauté.'

Au total huit élèves de la métropole ont été envoyés dans un territoire d'outre-mer et quinze sont venus de ces pays dans la métropole. Leur nombre sera plus important cette année comme sera plus important le nombre de jeunes gens qui aujourd'hui réfléchissent sur les problèmes posés par l'édification de cette vaste communauté qu'est l'Union française.

A Paris, la semaine a commencé par une conférence à la Sorbonne, prononcée par M. Hubert Deschamps, Directeur adjoint de la recherche scientifique à la France d'Outre-mer, à l'intention des professeurs. Le i $\varsigma$ mars, au Musée pédagogique, des délégations des lycées, collèges et écoles de Paris ont assisté à une conférence de Mme Malroux, suivie de la projection du film 'Paysans Noirs'.

\section{Un Centre permanent d'Art africain}

UN centre artisanal africain a été ouvert à Brazzaville où seront offerts non seulement les œuvres des étudiants et artisans de l'École des Arts et de l'artisanat de Brazzaville, mais également les produits de l'artisanat populaire spontané, particulièrement riche au Tchad.

L'École d'Art du Moyen-Congo en sera l'un des principaux exposants et fournisseurs, mais on parle également d'un important arrivage d'objets du 'Tchad et spécialement de très beaux tapis. Cette réalisation n'a été possible que grâce à l'effort accompli par l'École d'Art de Brazzaville. L'École d'Art compte actuellement 33 élèves, répartis en trois années, soit I I élèves par promotion. Elle comprend trois sections : une section de reliure et de maroquinerie, une section de sculpture sur bois et ivoire, une section de céramique-poterie.

Les ateliers, les tours et les fours de l'école sont en outre mis à la disposition d'une coopérative d'artisans anciens élèves qui comprend $\mathrm{I} 3$ associés, dont 9 sont céramistes. On s'est efforcé depuis la création de ce centre de respecter l'inspiration et le style propre de l'art africain, en aidant surtout ses élèves à améliorer leur technique et la qualité de leur production, en les défendant contre les facilités et plus encore contre les tentations du mercantilisme.

\section{Le développement des Sciences sociales et le Congo Belge}

L'importance d'une investigation méthodique en Afrique dans le champ des sciences sociales est admise partout. Dans le Congo Belge, l'Institut pour la recherche scientifique en Afrique Centrale, hautement conçu, a entrepris pendant plusieurs années une œuvre appréciée dans ce domaine. D'autres initiatives des Universités belges sont en train de se faire jour. L'Institut de Sociologie de l'Université de Liège entreprend d'établir un centre de recherches à Élisabethville, grâce à la collaboration du Centre d'études des problèmes sociaux indigènes (C.E.P.S.I.) et au bienveillant appui du Ministre des Colonies. Le développement de ce Centre, qui constituera une contribution de l'Université à l'œuvre africaine dans le domaine des sciences sociales, servira du même coup le développement des études sociologiques à Liège.

\section{Institute of Social and Economic Research, Rhodes University}

Mr. M. WILkEs has begun a two years' study of the conservative rural Xhosa (' red blanket people ') as part of the Border Regional Survey (see Africa, April 1956, p. 188). He is at present engaged on an intensive field-study among a rural community in the King Williamstown District.

Dr. D. H. Reader is entering the second stage of his field-work among urban Xhosa in the Native Locations of East London, and is collecting and analysing quantitative data with the help of African assistants. 